\title{
Recruiting TP53 to target chronic myeloid leukemia stem cells
}

\section{Steven Grant}

Virginia Commonwealth University, Richmond, VA, USA

E-mail: STEVEN GRANT - steven.grant@vcuhealth.org

doi:10.3324/haematol.2019.246306

T he problem of eradicating leukemic stem cells (LSC) in chronic myeloid leukemia (CML), as in the case of acute myelogenous leukemia (AML), has long been a therapeutic goal, particularly in relation to agents such as tyrosine kinase inhibitors (TKI), such as imatinib mesylate (IM), that target the oncogenic $\mathrm{BCR} / \mathrm{ABL}$ kinase. The central conundrum is that CML stem cells appear to be intrinsically resistant to TKI through a variety of mechanisms, including (but not restricted to) increased drug efflux pumps ${ }^{1}$ or persistence in a drug-resistant quiescent state. The failure of IM or newer-generation TKI to eradicate CML stem cells presumably underlies the development of drug resistance and/or progression to a more aggressive clinical course e.g. accelerated or blast-phase disease. This inevitable outcome (in transplant-ineligible patients) has prompted the search for new therapeutic strategies capable of eliminating the subset of TKI-resistant stem cells.

In the article by Carter et al., which appears in this edition of Haematologica, ${ }^{2}$ the authors expand upon their previous work investigating novel strategies specifically capable of targeting CML LSC. This group had previously shown that the BH3 mimetic Venetoclax (ABT-199), an agent now approved (in combination with hypomethylating agents) in older AML patients, targets LSC, and, when administered in conjunction with IM, effectively eradicated CML stem cells. ${ }^{3}$ This group had also shown that disrupting the function of MDM2 (e.g. with nutlin), a protein that binds to and inactivates TP $53,{ }^{4}$ also enhanced the activity of IM in a CML blast crisis model. The mechanism(s) by which activating TP53 might sensitize CML stem cells to IM remains to be fully elucidated, but very likely reflects induction of downstream TP53 pro-apoptotic effectors such as NOXA, PUMA, BAX, and BID. For example, NOXA is known to trigger degradation of anti-apoptotic proteins such as MCL- $1,{ }^{5}$ which have been shown to serve as a survival factor for leukemia stem cells. ${ }^{6}$

In the present study, the authors examined the effects of a newer MDM2 antagonist (DS-5272) on the sensitivity of CML stem cells to IM using an inducible stem cell promoter-driven CML murine model (Scl-tTa-BCR/ABL1). Employing CyTOF-based single-cell proteomics, they found that combined BCR/ABL1 and MDM2 inhibition resulted in the selective upregulation of NOXA and BAX in the CML-LSC population. Importantly, the combination strategy was effective in prolonging survival in this mouse model and in decreasing CML LSC frequency in secondary transplantations. The authors conclude that CML LSC may depend upon TP53 hyperactivation for survival, and that disruption of this process e.g. by MDM2 antagonism may restore TKI sensitivity in these cells. A schematic summary of these concepts is shown in Figure 1. According to this model, CML LSC exhibit rela- tive resistance to TKI, but high activity of TP53, the lethal effects of which are kept in check by MDM2. Disabling of the latter process, e.g. by MDM2 antagonists, results in increased expression of pro-apoptotic TP53-dependent proteins, e.g. NOXA and BAX, which lower the threshold for TKI-mediated cell death (Figure 1A). Activation of TP53 may also lead to downregulation of anti-apoptotic proteins such as MCL-1 indirectly through induction of NOXA. Alternatively, pro-apoptotic proteins such as BCL-2 may be disabled by small molecule BH3-mimetics such as ABT-199, analogously promoting TKI-induced cell death (Figure 1B). The net effect of these events is the selective eradication of CML LSC, an outcome unlikely to be accomplished with TKI alone.

If validated, these findings could have significant implications for the treatment of chronic phase CML by raising the possibility that concomitant administration of MDM2 antagonists with a TKI such as IM might, by targeting quiescent CML stem cells, delay or prevent the emergence of AP or BC. The success of this strategy will be contingent upon the presence of functioning TP53, as the results of earlier studies, as well as this present one, argue strongly that activation of this oncogene is essential for the beneficial actions of MDM2 antagonists. One implication of these findings is that early incorporation of MDM2 inhibitors into TKI-based therapies for CML may be necessary for optimal benefit. For example, in the case of AML, loss of functional TP53 occurs late in the disease and is associated with a particularly poor prognosis. Thus, early eradication of CML stem cells through such a TP53-based strategy may forestall or circumvent the emergence of aggressive clones that have lost functional TP53. Furthermore, while Carter et al. have previously described the capacity of the TKI/MDM2 antagonist strategy to target $\mathrm{BC}$ cells, ${ }^{8}$ the later this approach is applied, the greater the chance of the development of TP53-deficient cells that are resistant to its lethal activity.

One question that arises concerns the mechanism(s) by which addition of an MDM2 antagonist might enhance the activity of TKI against CML stem cells. As noted previously, CML LSC tend to be resistant to TKI because of several factors, including their quiescent state, as well as increased drug efflux in this cell population. ${ }^{9}$ To date, there is no evidence that MDM2 inhibitors can directly circumvent these mechanisms and as a consequence, restore TKI sensitivity. Instead, the former agents may primarily operate to modulate the apoptotic threshold e.g. by inducing NOXA, BAX, and potentially other TP53-dependent pro-apoptotic effectors. ${ }^{10}$ The ability of the combined TKI/MDM2 antagonist regimen to display superior LSC killing argues that this strategy acts, at least in part, to potentiate the ability of TKI to induce cell death rather than to overcome intrinsic TKI resistance in primitive leukemia progenitors. 


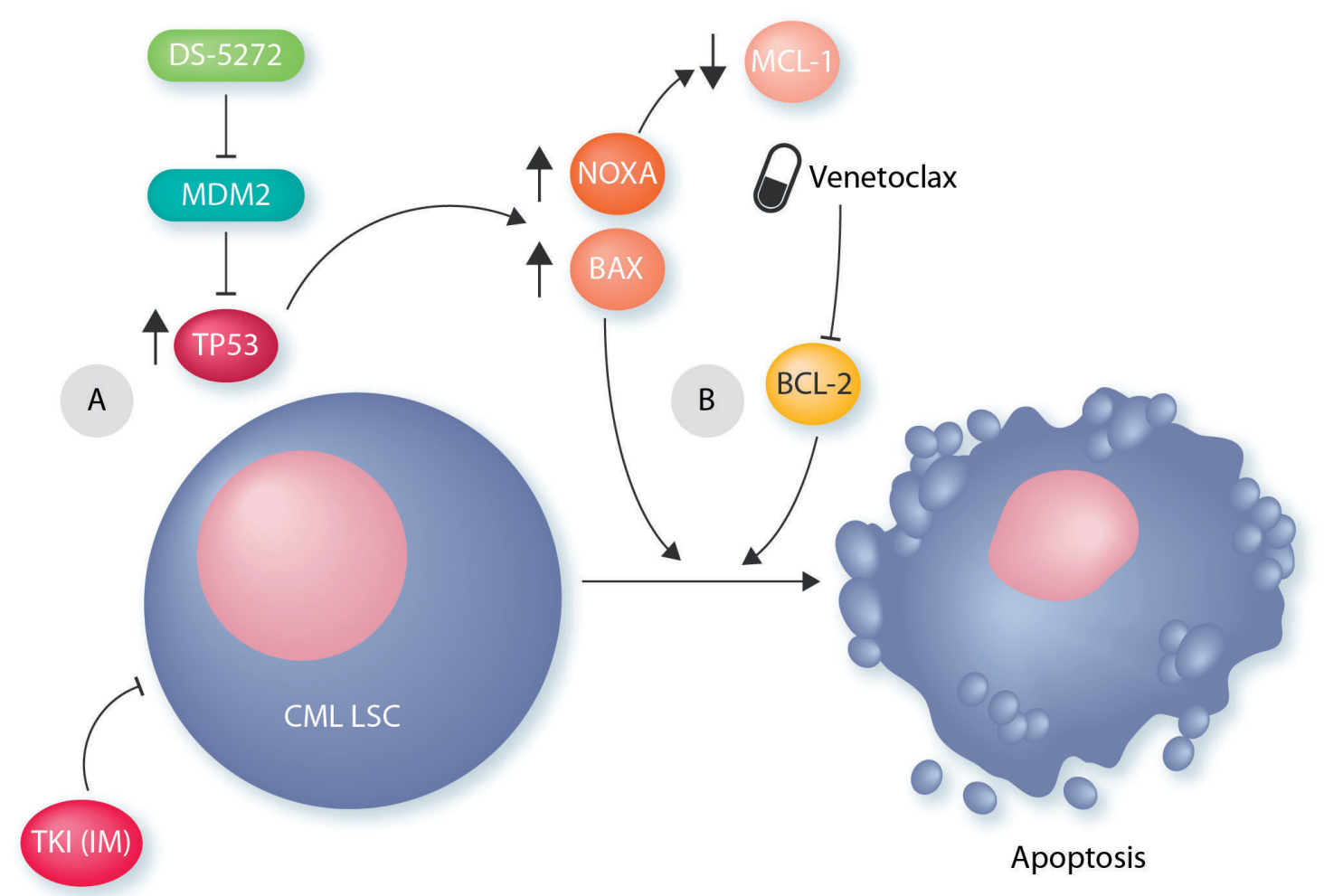

Figure 1. Chronic myeloid leukemia (CML) stem cells are resistant to tyrosine kinase inhibitors (TKI) due to multiple factors, including increased drug efflux. Combined treatment with a TKI and an inhibitor of MDM2 (e.g. OS-5272) leads to hyper-activation of TP53 (A). The latter event results in upregulation of multiple pro-apoptotic effectors including NOXA and PUMA, which trigger LSC apoptosis. This phenomenon may be enhanced by NOXA-mediated degradation of the anti-apoptotic protein MCL-1. Alternatively, disabling of the anti-apoptotic protein BCL-2 (B) e.g. by ABT-199 (Venetoclax) may analogously lower the threshold for TKI-induced cell death in the CML LSC subpopulation. Eradication of such stem cells by simultaneous targeting of oncogenic (BCR/ABL) and orthogonal (TP53) pathways may delay or prevent re-emergence of disease.

While the results of the Carter et al. study support the notion that an agent such as OS-5272 may potentiate the activity of a TKI like IM, they also lay a foundation for the development of a wealth of additional strategies capable of targeting CML LSC. For example, apart from the possibility of employing other MDM2 inhibitors, it would be of interest to assess interactions involving other agents that have been used to target BCR/ABL-expressing cells. These would include newer generation TKI such as the multikinase inhibitor ponatinib, which is active against BCR$\mathrm{ABL}^{+}$cells resistant to IM through the T315 mutation. ${ }^{11}$ An alternative candidate for combination with MDM2 inhibitors would be omacetaxine, a protein synthesis inhibitor which down-regulates BCR/ABL among other proteins, and which has shown significant activity against IM-resistant CML. ${ }^{12}$ Based upon the present results, there would seem to be a reasonable chance that MDM2 antagonist regimens incorporating these agents would also target LSC. In addition, as noted by the authors, the BCL-2 antagonist ABT-199 is known to target LSC, ${ }^{13}$ and has also been shown to increase TKI activity against primitive LSC. ${ }^{3}$ If tolerable, adding ABT-199 to the MDM2 antagonist/IM regimen may prove to be particularly effective in eliminating the LSC population. Aside from this approach, recent attention has focused on the development of MCL1 antagonists as anti-leukemic agents ${ }^{14}$ in part due to evidence that MCL-1 is required by CML LSC for survival. ${ }^{15}$
In view of these considerations, the notion of using a clinically relevant MCL-1 antagonist to further enhance the activity of an MDM2 antagonist/TKI regimen against CML LSC appears worthy of investigation.

In summary, the present report argues that, in CML, LSC exhibit a potentially selective activation of TP53 which can be exploited through the use of MDM2 antagonists, and that this phenomenon can act in concert with TKI inhibitors to trigger cell death in this difficult to eradicate leukemic cell subpopulation. Whereas in the past, attention focused on the identification of novel TKI able to overcome mutational forms of resistance, more current approaches are based on the development of strategies designed to disrupt orthogonal, non-oncogenic pathways ${ }^{16}{ }^{16}$ including those related to TP53. However, whether such dual-targeting strategies will prove capable of eliminating primitive stem cells has not yet been definitively established. The results presented here strongly support the concept of simultaneous targeting of oncogenic drivers (e.g. BCR/ABL) and orthogonal pathways (e.g. TP53) to eradicate these cells, at least in the case of CML. Given the large array of targeted agents that are now clinically available, including MDM2 inhibitors and modulators of the apoptotic response, LSC-directed therapy in CML and related disorders is currently underway. Determining whether such novel strategies will live up to their pre-clinical promise is only a matter of time. 


\section{References}

1. Engler JR, Frede A, Saunders VA, Zannettino AC, Hughes TP, White DL. Chronic myeloid leukemia CD34+ cells have reduced uptake of imatinib due to low OCT-1 activity. Leukemia. 2010;24(4):765-770.

2. Carter BZ, Mak PY, Mu H, et al. Combined inhibition of MDM2 and $\mathrm{Bcr}-\mathrm{Abl}$ tyrosine kinase targets chronic myeloid leukemia stem/progenitor cells in a murine model. Haematologica. 2020;105(5):12741284.

3. Carter BZ, Mak PY, Mu H, et al. Combined targeting of BCL-2 and BCR-ABL tyrosine kinase eradicates chronic myeloid leukemia stem cells. Sci Transl Med. 2016;8(355):355ra117.

4. Lau LM, Nugent JK, Zhao X, Irwin MS. HDM2 antagonist Nutlin-3 disrupts p73-HDM2 binding and enhances p73 function. Oncogene. 2008;27(7):997-1003.

5. Willis SN, Chen L, Dewson G, et al. Proapoptotic Bak is sequestered by $\mathrm{Mcl}-1$ and $\mathrm{Bcl}-\mathrm{xL}$, but not Bcl-2, until displaced by $\mathrm{BH} 3$-only proteins. Genes Dev. 2005;19(11):1294-1305

6. Yoshimoto G, Miyamoto T, Jabbarzadeh-Tabrizi S, et al. FLT3-ITD up-regulates MCL-1 to promote survival of stem cells in acute myeloid leukemia via FLT3-ITD-specific STAT5 activation. Blood. 2009;114(24):5034-5043.

7. Hunter AM, Sallman DA. Current status and new treatment approaches in TP53 mutated AML. Best Pract Res Clin Haematol. 2019;32(2):134-144.

8. Carter BZ, Mak PY, Mak DH, et al. Synergistic effects of p53 activation via MDM2 inhibition in combination with inhibition of $\mathrm{Bcl}-2$ or $\mathrm{Bcr}-\mathrm{Abl}$ in CD34+ proliferating and quiescent chronic myeloid leukemia blast crisis cells. Oncotarget. 2015;6(31):30487-30499.

9. Inoue A, Kobayashi CI, Shinohara H, et al. Chronic myeloid leukemia stem cells and molecular target therapies for overcoming resistance and disease persistence. Int J Hematol. 2018;108(4):365370 .

10. Peterson LF, Mitrikeska E, Giannola D, et al. p53 stabilization induces apoptosis in chronic myeloid leukemia blast crisis cells. Leukemia. 2011;25(5):761-769.

11. Pavlovsky C, Chan O, Talati C, Pinilla-Ibarz J. Ponatinib in the treatment of chronic myeloid leukemia and philadelphia chromosome positive acute lymphoblastic leukemia. Future Oncol. 2019;15(3): 257-269.

12. Novotny L, Al-Tannak NF, Hunakova L. Protein synthesis inhibitors of natural origin for CML therapy: semisynthetic homoharringtonine (Omacetaxine mepesuccinate). Neoplasma. 2016;63(4):495-503.

13. Mattes K, Gerritsen M, Folkerts H, et al. CD34+ acute myeloid leukemia cells with low levels of reactive oxygen species show increased expression of stemness-genes and can be targeted by the BCL2 inhibitor Venetoclax. Haematologica. 2019 Nov 14. [Epub ahead of print]

14. Wang Q, Hao S. A-1210477, a selective MCL-1 inhibitor, overcomes ABT-737 resistance in AML. Oncol Lett. 2019;18(5):5481-5489.

15. Allan EK, Holyoake TL, Craig AR, Jørgensen HG. Omacetaxine may have a role in chronic myeloid leukaemia eradication through downregulation of Mcl-1 and induction of apoptosis in stem/progenitor cells. Leukemia. 2011;25(6):985-994.

16. Hanahan D, Weinberg RA. Hallmarks of cancer: the next generation. Cell. 2011;144(5):646-674.

\title{
Role of Meningioma 1 for maintaining the transformed state in MLL-rearranged acute myeloid leukemia: potential for therapeutic intervention?
}

\author{
Juerg Schwaller \\ University Children's Hospital beider Basel (UKBB), Department of Biomedicine (DBM), University of Basel, Switzerland \\ E-mail: JUERG SCHWALLER - j.schwaller@unibas.ch
}

doi:10.3324/haematol.2019.246348

$\mathrm{M}$ eningioma 1 (MN1) was cloned from a balanced chromosomal translocation in a meningioma as open reading frame encoding for a protein of 1,319 amino acids containing several proline and histidine-rich domains, acting as a transcriptional activator necessary for normal development of the bones of the skull. ${ }^{1,2}$ Several studies found mutations or aberrant expression of $\mathrm{MN1}$ in various hematologic malignancies. Characterization of a $\mathrm{t}(12 ; 22)(\mathrm{p} 13 ; \mathrm{q} 11)$ chromosomal translocation associated with myeloproliferative disorders revealed a fusion between MN1 and the ETSfamily transcription factor ETV6 (a.k.a. TEL). ${ }^{3}$ A fusion of $\mathrm{MN1}$ to Friend leukemia virus integration 1 (FLI1) has been shown to be a rare transforming oncogene in acute megakaryoblastic leukemia (AMKL). ${ }^{4}$ Aberrant high expression of $\mathrm{MN1}$ was reported in acute myeloid leukemia (AML) with inv(16) leading to the core-binding factor fusion CBFB-MYH11. ${ }^{5}$ Clinical studies proposed that high MN1 transcript levels could be used as prognostic marker in cytogenetically-normal (CN) AML. ${ }^{6}$ Functional studies in mice demonstrated the oncogenic potential of aberrant MN1 expression. Retroviral MN1 overexpression in murine bone marrow (BM) hematopoietic stem and progenitor cells (HSPC) followed by transplantation rapidly induced a lethal AML in mice. ${ }^{7}$ Aberrant MN1 expression due to retroviral insertion was shown to act as collaborative oncogenic event in acute leukemia induction by the MLL-ENL or the MLL-AF9 fusion gene, respectively., ${ }^{8,9}$ More recent work suggested that gene expression programs associated with MN1-mediated transformation of hematopoietic cells are controlled by the H3K4 and H3K79 histone methyltransferases MLL1 and DOT1L, respectively. ${ }^{10}$ Collectively, these studies indicated that aberrant MN1 expression contributes to malignant transformation of hematopoietic cells towards AML; however, its role in the maintenance of the transformed state remained poorly understood.

In a study published in this issue of Haematologica, Sharma et al. functionally addressed the role of MN1 in the maintenance of AML induced by MLL fusion oncogenes. ${ }^{11}$ They used Crisper/CAS9 to ablate MN1 in several murine AML lines, including cells transformed by retroviral overexpression of the MLL-AF9 fusion (rMLL-AF9), and in human THP1 and MV4;11 AML cell lines carrying the MLL-AF9 and MLL-AF4 fusions, respectively. They found that inactivation of MN1 impaired the clonogenic activity and proliferation associated with impaired cell cycle progression, and increased differentiation and apoptosis of murine rMLL-AF9 AML cells. Loss of MN1 also 